\title{
Enfermedad bullosa como complicación pulmonar en la convalecencia de COVID-19
}

\author{
Bullous disease as a pulmonary complication \\ in the convalescence of COVID-19
}

\author{
José Antonio Rodríguez Blanco ${ }^{1}$, Jesús Daniel Rodríguez Blanco ${ }^{2}$, Jonathan José Rodríguez \\ Blanco $^{3}$, María Cristina Martínez Ávila ${ }^{4}$, María Camila Acuña Caballero ${ }^{5}$, Carlos Emilio \\ REMOLINA. ${ }^{1}$
}

\section{Resumen}

La infección por SARS-CoV-2 comenzó en Wuhan, China, a principios del año 2019 y se ha extendido rápidamente por todo el mundo, convirtiéndose en pandemia de acuerdo con la Organización Mundial de la Salud (OMS) el 11 de marzo de 2020. Esta enfermedad ha presentado múltiples manifestaciones que varían desde un resfriado común hasta un síndrome de dificultad respiratoria aguda (SDRA) grave con complicaciones en pacientes críticos o durante la fase aguda. Hasta la fecha, las complicaciones pulmonares tardías y las secuelas no son del todo conocidas, sin embargo, se han asociado a la gravedad de la enfermedad. Se presenta el primer caso en Colombia de una enfermedad bullosa gigante ocurrido en una paciente femenina de 32 años durante la convalecencia de la infección por COVID-19, que requirió resección quirúrgica. Se hace necesario resaltar la importancia del seguimiento de la población que se ha contagiado de este virus y mantener un alto grado de sospecha clínica en la búsqueda de complicaciones en individuos que se han recuperado de la infección, con el fin de aprender cada día más sobre el comportamiento, las complicaciones y las secuelas de esta enfermedad en aras de dar un manejo óptimo y oportuno.

Palabras clave: coronavirus, COVID-19, neumonía, bulla, complicación, tomografía de tórax.

\begin{abstract}
COVID-19 infection began in Wuhan, China, and has spread rapidly all over the world, becoming pandemic according WHO on March 11, 2020. This disease has presented multiple manifestations that vary from a common cold to a severe acute respiratory distress syndrome with complications in critically ill patients or during the acute phase. To date, late pulmonary complications and sequelae remain unknown; however, they have been associated with the severity of the disease. We report the first case in Colombia of a giant bullous disease that required surgical resection, occurred in a 32-year-old female patient during convalescence from COVID 19 infection. It is necessary to highlight the importance of monitoring the population that has been infected by this virus a high degree of suspicion should be maintained in searching for causes of chronic lung disease in patients who have recovered from the infection, in order to learn every day more about the behavior, complications and sequelae that this disease causes in order to provide optimal and timely management.
\end{abstract}

Keywords: Coronavirus; COVID-19; Pneumonia; Bullae; Complication; Tomography.
${ }^{1}$ Médico especialista en Cirugía y $\mathrm{Fe}$ llow en Cirugía de Tórax, Clínica Gestión Salud IPS. Cartagena, Colombia.

${ }^{2}$ Médico, Universidad del Sinú, sede Cartagena. Unidad de Cuidados Intensivos, Nuevo Hospital Bocagrande. Cartagena, Colombia.

${ }^{3}$ Médico especialista en Anestesiología, Unidad de Cuidados Intensivos, Nuevo Hospital Bocagrande. Cartagena, Colombia.

${ }^{4}$ Médico especialista en Epidemiología Clínica, Unidad de Cuidados Intensivos, Nuevo Hospital Bocagrande. Cartagena, Colombia.

${ }^{5}$ Médico, Universidad del Sinú, sede Cartagena. Unidad de Cuidados Intensivos, CMI Internacional. Barranquilla, Colombia.

Autor de correspondencia

Jesús Daniel Rodríguez Blanco

Correo electrónico:

jesusdanielrb@gmail.com

Recibido: 20/08/2020

Aceptado: 10/10/2020 


\section{Introducción}

Las bullas o ampollas pulmonares se definen como espacios aéreos mayores de $1 \mathrm{~cm}$ de diámetro en forma de globo, que carecen de pared epitelial, formadas a partir de tejido pulmonar estructuralmente dañado tras la destrucción, la dilatación y la confluencia de espacios aéreos distales a los bronquiolos terminales debido a una variedad de etiologías (1). La enfermedad pulmonar bullosa hace referencia a la presencia de bullas en un pulmón sin alteraciones, se presenta en el $20 \%$ de los casos; mientras que el enfisema ampolloso que puede verse en el parénquima pulmonar enfisematoso es una enfermedad localizada en el $80 \%$ de los casos (2). La tomografía nos permite valorar la localización, la extensión y la distribución de las bullas y sus complicaciones subyacentes (3). Con frecuencia se asocia a distintas patologías pulmonares, como enfermedad pulmonar obstructiva crónica (EPOC), bronquitis crónica, insuficiencia cardíaca, así como en pacientes jóvenes con antecedentes de tabaquismo (4).

La presentación clínica de los pacientes con bullas pulmonares es muy variada, desde hallazgos incidentales de pequeñas ampollas en pacientes asintomáticos con función pulmonar normal hasta el síndrome del pulmón evanescente, el cual se manifiesta clínicamente con disnea severa, insuficiencia respiratoria o cor pulmonale debido a la pérdida de parénquima pulmonar funcional debido a la compresión por las bullas (5). Estas pueden romperse y producir fugas de aire que pueden o no ir a la cavidad pleural, lo que da lugar a complicaciones que incluyen neumotórax, neumatocele, neumomediastino, empiema, hemoptsis o sangrados, fístulas broncopleurales, disnea crónica, en casos excepcionales patologías oncológicas, entre otras (6).

El perfil clínico de la infección por SARS-CoV-2 varía desde una infección asintomática hasta una enfermedad crítica, que incluye comúnmente tos, fiebre, mialgias, síntomas gastrointestinales y anosmia (7). La fase aguda de la enfermedad es compatible con muchas complicaciones graves que comprometen la vida, como insuficiencia respiratoria, SDRA, fibrosis pulmonar, arritmias, lesión cardíaca aguda, choque séptico, eventos tromboembólicos y complicaciones inflamatorias (8). Muchos pacientes con COVID-19 han sido tratados con ventilación mecánica invasiva, lo cual puede causar complicaciones. Inusualmente se han reportado casos de barotrauma, enfisema subcutáneo, neumotórax, neumomediastino espontáneo, cavitaciones y bullas pulmonares, hasta el momento todas complicaciones bien conocidas de la ventilación mecánica; no obstante, no se descartan que estén asociadas con el curso natural de la neumonía por COVID-19 (9).

Se relata el caso clínico de una paciente adulta sin comorbilidades previas, que consultó por disnea progresiva tres meses después de una infección leve por COVID-19. En las imágenes del tórax se evidenció una bulla gigante que se atribuyó al antecedente de la infección viral. En los estudios previos de la paciente no había alteraciones radiológicas similares. Esta paciente requirió un manejo quirúrgico, el cual resultó exitoso por el servicio de cirugía de tórax. Se discute el caso clínico, las intervenciones y su desenlace.

\section{Descripción del caso}

Paciente femenina de 32 años, residente en la ciudad de Cartagena, Colombia, con antecedentes de bypass gástrico hace 8 años y osteosíntesis de peroné hace 11 años, consulta el 29 de marzo de 2020 por un cuadro clínico de 1 semana de evolución consistente en sensación de disnea con nexo epidemiológico positivo para COVID-19. Niega otros antecedentes médicos de relevancia, no fumadora y sin enfermedad pulmonar previa. A su ingreso los signos vitales están dentro de los parámetros normales, y su saturación de oxígeno $\left(\mathrm{SatO}_{2}\right)$ ambiente es mayor a $95 \%$. Se realizan paraclínicos, radiografía de tórax y gasometría arterial que resultan sin alteraciones, con una prueba rápida de inmunoglobulina (Ig) $\mathrm{G}$ e Ig M positiva para SARS-CoV-2, por lo que se consideró como una infección no complicada por COVID-19. Ante la estabilidad clínica se da egreso hospitalario para manejo ambulatorio, medidas de aislamiento con recomendaciones y se indican los signos de alarma para volver a consultar.

Pasados 3 meses de la infección por COVID-19 la paciente consulta nuevamente a la institución por un cuadro de dolor torácico tipo pleurítico derecho de inicio súbito, asociado con el aumento de la disnea y un deterioro de la clase funcional. Los signos vitales se encuentran sin alteraciones: presión arterial (PA) de 116/74 mm Hg, frecuencia cardíaca (FC) de 82 latidos por minuto (lpm), frecuencia respiratoria (FR) de 16 res- 
piraciones por minuto (rpm), $\mathrm{SatO}_{2} 97 \%$ ambiente, afebril, Escala de Coma de Glasgow de 15/15. Al examen físico se encontró un buen patrón respiratorio, sin signos de dificultad respiratoria ni signos clínicos de insuficiencia cardíaca o ingurgitación yugular; en la auscultación cardiopulmonar se encontró una disminución del murmullo vesicular en la base pulmonar derecha, con una percusión resonante sin agregados pulmonares. No se encontraron signos de trombosis venosa profunda. En los paraclínicos no se encontró leucocitosis, neutrofilia, anemia ni trombocitopenia, las funciones hepática y renal conservadas, sin alteraciones hidroelectrolíticas y gasometría arterial sin trastornos de la oxigenación. Además, se le realiza una serología institucional RTPCR para SARS-CoV-2, la cual fue negativa.

Se indica una tomografía de tórax de alta resolución, donde se aprecia una gran bulla basal derecha de $99 \mathrm{~mm}^{3}$ de paredes muy finas, sin ruptura ni signos de sobreinfección (Figura 1). Es de resaltar que la paciente tiene una radiografía de tórax previa, la cual no evidencia la presencia de esta lesión pulmonar (Figura 2). Ante los hallazgos tomográficos se hospitaliza a la paciente en la sala general y se realizan estudios complementarios para evaluar la etiología y los diagnósticos diferenciales. En la espirometría se encontró un patrón restrictivo leve, el ecocardiograma transtorácico no mostró signos indirectos de tromboembolismo pulmonar ni alteraciones en las cavidades derechas, y una fracción de eyección preservada del ventrículo izquierdo (FEVI) de $65 \%$.

La paciente persiste sintomática, por lo que es valorada por el área de cirugía de tórax, donde se considera que cumple los criterios para la realización de una cirugía debido a la presencia de una compresión del parénquima pulmonar sano y un compromiso de más de un tercio del hemitórax derecho; por tanto, se programa para la resección de las bullas pulmonares por videotoracoscopia más pleurectomía parietal por la misma vía. El procedimiento quirúrgico se realiza sin complicaciones, y se traslada a la paciente a la unidad de cuidados intensivos (UCI) en el posoperatorio, con evolución satisfactoria. El drenaje torácico no presentó escape de aire y el débito de líquido fue bajo; la radiografía de tórax de control mostró una expansión pulmonar completa sin cámaras de neumotórax ni hidrotórax (Figura 3), por lo que se retiró el tubo de tórax y se le dio el alta hospitalaria. En las consultas de seguimiento refiere una mejoría de los síntomas respiratorios con buena capacidad funcional, y no ha presentado complicaciones ni reincidencia de los síntomas iniciales.
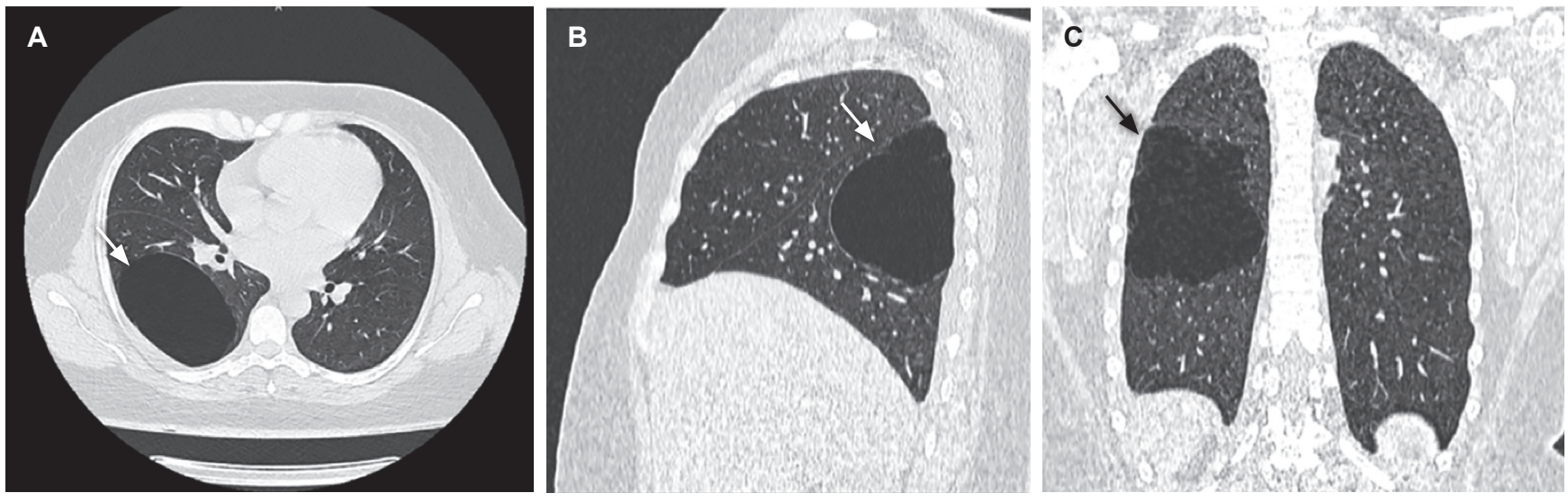

Figura 1. A, B y C. Tomografía axial computarizada de tórax que evidencia una gran bulla pulmonar, la cual compromete más de 1/3 del hemitórax derecho, localizada en el lóbulo inferior, sin neumotórax ni patrón de neumonía viral. 


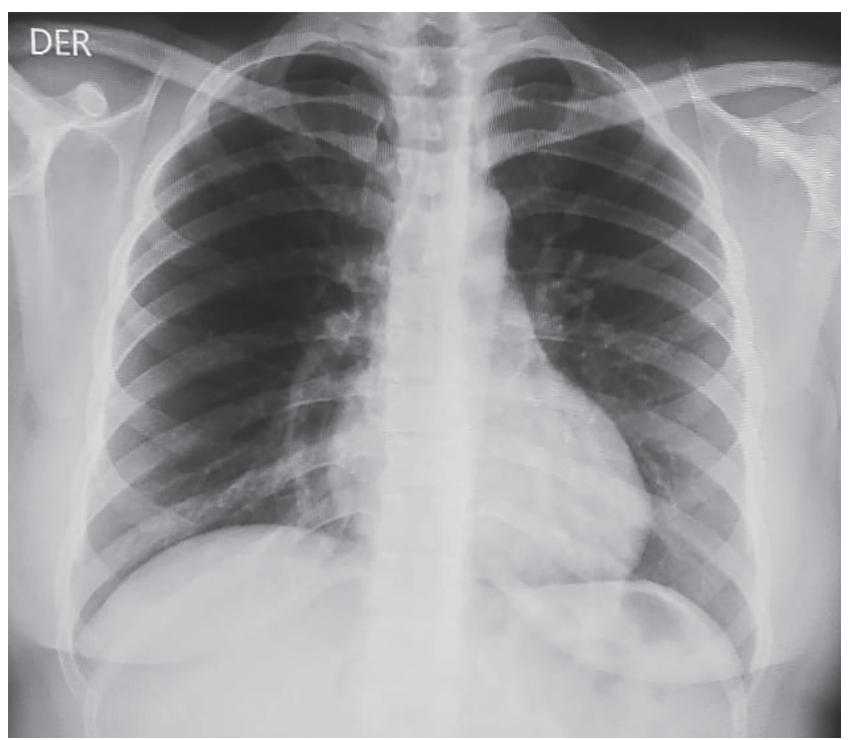

Figura 2. Radiografía de tórax previa a la infección por COVID-19. No se evidencian alteraciones radiológicas.

\section{Discusión y conclusiones}

El dolor torácico pleurítico de inicio súbito asociado con la sensación de disnea en un paciente con COVID-19 puede atribuirse a la progresión de la enfermedad; sin embargo, la presentación unilateral del caso obliga a la investigación de complicaciones ya conocidas como la embolia pulmonar y el neumotórax por el estado protrombótico e inflamatorio secundario $(10,11)$. Una tomografía computarizada de tórax en esta paciente mostró una bulla gigante en el pulmón derecho sin ruptura o neumotórax. Tres meses antes (Figura 2) no se observaban cambios bullosos en la radiografía.

Por lo general, las bullas pulmonares se pueden presentar como una enfermedad primaria o secundaria a enfisema o fibrosis, $\mathrm{y} / \mathrm{o}$ los pacientes pueden tener una predisposición genética a ellas o factores de riesgo como tabaquismo $(4,12)$, enfermedad neoplásica pulmonar asociada (13), <20 años (14), traumas recientes, historia de enfermedad cardiopulmonar, antecedentes de intubaciones o ventilación mecánica, uso de drogas (15) y, ahora, el COVID-19 (16), lo que resalta así la gravedad y la complejidad de esta enfermedad emergente.

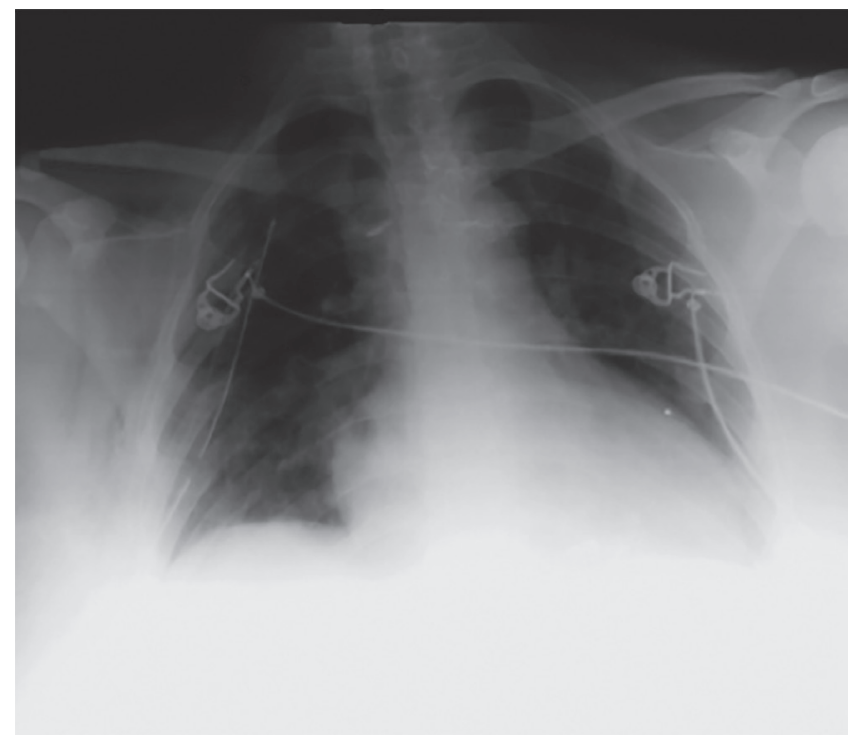

Figura 3. Radiografía de tórax portátil posoperatoria donde se aprecia una buena expansibilidad pulmonar, tubo a tórax derecho, sin neumotórax.

En el período posinfección o convaleciente de COVID-19 se ha informado que los pacientes presentan el síndrome pos-COVID, que va desde adinamia hasta complicaciones más críticas como accidente cerebrovascular, insuficiencia renal, miocarditis y fibrosis pulmonar, los cuales están relacionados con las comorbilidades, el estado de hipercoagulabilidad y la gravedad de la enfermedad (17).

Dentro de la literatura se han descrito algunos casos de pacientes con neumonía por COVID-19 sin antecedentes de enfermedad pulmonar crónica ni tabaquismo, sometidos a presión positiva continua en la vía aérea durante el período crítico de la enfermedad, con resultados satisfactorios en la evolución y que, al final, terminaron desarrollando una enfermedad ampollosa pulmonar grave unilateral, neumatocele y neumotórax secundario, lo que conllevó la readmisión dentro de las 2 semanas posteriores al alta (12). Asimismo, se presentaron otros casos con neumotórax espontáneo precedidos de una gran bulla asociados con la infección por SARS-CoV-2 durante el curso de la enfermedad ( 9 , 18). Es de mencionar que, en otros tipos de neumonitis 
viral, como las originadas por el virus de la influenza, el sarampión, el hantavirus y el citomegalovirus, que también se asocian con opacificación y consolidación de vidrio esmerilado, se han encontrado casos de neumotórax espontáneo, bullas y complicaciones como fibrosis pulmonar y cambios intersticiales $(19,20)$.

Hasta el momento no se han reportado casos con complicaciones severas en pacientes con síntomas leves, como el caso descrito. Los informes de neumotórax espontáneo, neumotórax a tensión o neumomediastino como complicación pulmonar tardía del COVID-19 en ausencia de enfermedad pulmonar subyacente o barotrauma debido a la ventilación mecánica son raros, y la formación de bullas no se ha informado en Colombia. Además, el paciente no requirió oxigenoterapia ni presiones positivas en la vía aérea previo a la aparición de la bulla. Es por esto por lo que, tras descartar otras causas o etiologías en este caso en específico, la aparición de enfermedad bullosa se atribuyó a la infección por COVID-19.

Aún se desconoce la fisiopatología de los cambios quísticos y la formación de ampollas en el COVID-19, pero se cree que el daño alveolar causado por el SARSCoV-2 promueve una destrucción del tejido alveolar que da como resultado la formación de bullas, lo que aumenta el riesgo de neumotórax (7). En este proceso se cree que la tormenta inflamatoria también desempeña un papel importante (21). Se necesitan más estudios para evaluar la enfermedad pulmonar a largo plazo (22).

Es de destacar el papel tan importante que desempeñan las imágenes diagnósticas en esta enfermedad no solo en el curso de la enfermedad, sino también durante su seguimiento para valorar la aparición de complicaciones como fibrosis pulmonar, compromiso intersticial, neumotórax, la formación de ampollas y otros cambios estructurales. En este caso, la tomografía computarizada sugirió una enfermedad ampollosa generalizada, y la paciente recibió tratamiento quirúrgico con un desenlace exitoso.

En resumen, durante esta época de pandemia por COVID-19 encontramos una enfermedad nueva con una gran variedad de presentaciones clínicas y complicaciones tanto a corto como a mediano y largo plazo.
En este caso, se destaca la inusual complicación (potencialmente grave) de la aparición de una bulla gigante en una paciente previamente sana sin factores de riesgo particulares para esta enfermedad justo después de la infección por COVID-19 con síntomas leves, situación con una fisiopatología aún no tan clara.

\section{Conflicto de intereses}

Los autores declaran no tener ningún conflicto de intereses. Se firmó el consentimiento informado para la realización y la divulgación del artículo.

\section{Referencias}

1. Erne BV, Graff M, Klemm W, Danzl JG, Leschber G. Bulla in the lung. Lancet. 2012;380(9849):1280. doi: 10.1016/S01406736(12)60690-4

2. Fishman A, Grippi M, Elias J, et al. Fishman's pulmonary diseases and disorders. 5. ${ }^{\text {a }}$ edición. Nueva York: McGraw-Hill Education; 2015. p. 1735-58.

3. Hansell D, Armstrong, Lynch, McAdams, et al. Tórax: diagnóstico radiológico. Editorial Marbán; 2018. p. 673-74.

4. Fraser RG, Pare JAP. Diseases of the airways: Bullous diseases of the lungs. 3. ${ }^{\text {a }}$ edición. Filadelfia: W.B. Saunders; 2017. p. 1420-31.

5. Klingman RR, Angelillo VA, DeMeester TR. Cystic and bullous lung disease. Ann Thorac Surg. 1991;52(3):576-80. doi: 10.1016/0003-4975(91)90939-n

6. Greenberg JA, Singhal S, Kaiser LR. Giant bullous lung disease: evaluation, selection, techniques, and outcomes. Chest Surg Clin N Am. 2003;13(4):631-49. doi: 10.1016/s10523359(03)00095-4

7. Janssen ML, van Manen MJG, Cretier SE, et al. Pneumothorax in patients with prior or current COVID-19 pneumonia. Respir Med Case Rep. 2020;31:101187. doi: 10.1016/j. rmcr.2020.101187

8. Bilal M, Khan MI, Nazir MS, Ahmed I, Iqbal HMN. Coronaviruses and COVID-19 - Complications and Lessons Learned for the Future. J Pure Appl Microbiol. 2020;14(suppl 1):72531. doi: 10.22207/jpam.14.spl1.09

9. Sun R, Liu H, Wang X. Mediastinal Emphysema, Giant Bu1la, and Pneumothorax Developed during the Course of COVID-19 Pneumonia. Korean J Radiol. 2020;21(5):541-44. doi: 10.3348/kjr.2020.0180

10. Lorenzo C, Francesca B, Francesco P, Elena C, Luca S, Paolo $\mathrm{S}$. Acute pulmonary embolism in COVID-19 related hypercoagulability. J Thromb Thrombolysis. 2020;50(1):223-26. doi: 10.1007/s11239-020-02160-1

11. Léonard-Lorant I, Delabranche X, Séverac F, Helms J, Pauzet C, Collange O, et al. Acute Pulmonary Embolism in Patients 
with COVID-19 at CT Angiography and Relationship to d-Dimer Levels. Radiology. 2020;296(3):E189-91. doi: 10.1148/ radiol.2020201561

12. Berhane S, Tabor A, Sahu A, Singh A. Development of bullous lung disease in a patient with severe COVID-19 pneumonitis. BMJ Case Rep. 2020;13(10):e237455. doi: 10.1136/ bcr-2020-237455

13. Aljehani Y, Almutairi M, Alreshaid F, El-Bawab H. Lung carcinoma within a lung bulla wall: literature review and case report. Asian Cardiovasc Thorac Ann. 2020;28(1):39-44. doi: $10.1177 / 0218492319896514$

14. Tsuboshima K, Matoba Y, Wakahara T, Maniwa Y. Natural history of bulla neogenesis for primary spontaneous pneumothorax: a propensity score analysis. Gen Thorac Cardiovasc Surg. 2019;67(5):464-469. doi: 10.1007/s11748-018-1046-3

15. Ahluwalia AS, Qarni T, Narula N, Sadiq W, Chalhoub MN. Bilateral pneumothorax as possible atypical presentation of coronavirus disease 2019 (COVID-19). Respir Med Case Reports. 2020;31:101217. doi: 10.1016/j.rmcr.2020.101217

16. Shi H, Han X, Jiang N, Cao Y, Alwalid O, Gu J, et al. Radiological findings from 81 patients with COVID-19 pneumonia in Wuhan, China: a descriptive study. The Lancet Infectious diseases. 2020;20(4):P425-34. doi: 10.1016/ S1473-3099(20)30086-4
17. Kamal M, Abo Omirah M, Hussein A, Saeed H. Assessment and characterisation of post-COVID-19 manifestations. Int J Clin Pract. 2020:e13746. doi: 10.1111/ijcp.13746

18. Lei P, Mao J, Wang P. Spontaneous Pneumomediastinum in a Patient with Coronavirus Disease 2019 Pneumonia and the Possible Underlying Mechanism. Korean J Radiol. 2020;21(7):929-30. doi: 10.3348/kjr.2020.0426

19. Chen J, Wu J, Hao S, Yang M, Lu X, Chen X, et al. Long term outcomes in survivors of epidemic Influenza A (H7N9) virus infection. Sci Rep. 2017;7(1):17275. doi: 10.1038/s41598017-17497-6

20. Bor C, Demirağ K, Uyar M, Cankayalı I, Moral AR. Recurrent Spontaneous Pneumothorax during the Recovery Phase of ARDS Due to H1N1 Infection. Balkan Med J. 2013;30(1):123-5. doi: 10.5152/balkanmedj.2012.086

21. Wang D, Hu B, Hu C, Zhu F, Liu X, Zhang J, et al. Clinical Characteristics of 138 Hospitalized Patients With 2019 Novel Coronavirus-Infected Pneumonia in Wuhan, China. JAMA. 2020;323(11):1061-69. doi: 10.1001/jama.2020.1585

22. Yasukawa K, Vamadevan A, Rollins R. Bulla Formation and Tension Pneumothorax in a Patient with COVID-19. Am J Trop Med Hyg. 2020;103(3):943-44. doi: 10.4269/ajtmh.20-0736 